Eva Heilmann, Claudia Gregoriano, Yannick Wirz, Charles-Edouard Luyt, Michel Wolff, Jean Chastre, Florence Tubach, Mirjam Christ-Crain, Lila Bouadma, Djillali Annane, Pierre Damas, Kristina B. Kristoffersen, Carolina F. Oliveira, Daiana Stolz, Michael Tamm, Evelien de Jong, Konrad Reinhart, Yahya Shehabi, Alessia Verduri, Vandack Nobre, Maarten Nijsten, Dylan W. deLange, Jos A.H. van Oers, Albertus Beishuizen, Armand R.J. Girbes, Beat Mueller and Philipp Schuetz*

\title{
Association of kidney function with effectiveness of procalcitonin-guided antibiotic treatment: a patient-level meta-analysis from randomized controlled trials
}

https://doi.org/10.1515/cclm-2020-0931

Received June 17, 2020; accepted August 31, 2020; published online September 28, 2020

\begin{abstract}
Objectives: Patients with impaired kidney function have a significantly slower decrease of procalcitonin (PCT) levels during infection. Our aim was to study PCT-guided
\end{abstract}

antibiotic stewardship and clinical outcomes in patients with impairments of kidney function as assessed by creatinine levels measured upon hospital admission.

Methods: We pooled and analyzed individual data from 15 randomized controlled trials who were randomly assigned to receive antibiotic therapy based on a PCT-algorithms or based on standard of care. We stratified patients on the initial glomerular filtration rate (GFR, $\mathrm{ml} / \mathrm{min} / 1.73 \mathrm{~m}^{2}$ ) in three groups (GFR >90 [chronic kidney disease; CKD 1], GFR
*Corresponding author: Prof. Philipp Schuetz, MD, University Department of Medicine, Kantonsspital Aarau, Tellstr., 5001 Aarau, Switzerland; and Faculty of Medicine, University of Basel, Basel, Switzerland, E-mail: schuetzph@gmail.com Eva Heilmann, Claudia Gregoriano and Yannick Wirz, Medical University Department, Kantonsspital Aarau, Aarau, Switzerland Charles-Edouard Luyt, Service de Médecine Intensive Réanimation, Sorbonne Université, Assistance Publique-Hôpitaux de Paris (AP-HP), Paris, France

Michel Wolff, Jean Chastre and Lila Bouadma, Service de Réanimation Médicale, Université Paris 7-Denis-Diderot, Assistance PubliqueHôpitaux de Paris (AP-HP), Paris, France

Florence Tubach, Département d'Epidémiologie Biostatistique et Recherche Clinique, AP-HP, Hôpitaux Universitaires Paris Nord Val de Seine, Paris, France

Mirjam Christ-Crain, Division of Endocrinology, Diabetology and Clinical Nutrition, University Hospital Basel, Basel, Switzerland Djillali Annane, Department of Critical Care, Hyperbaric Medicine and Home Respiratory Unit, Center for Neuromuscular Diseases, Raymond Poincaré Hospital (AP-HP), Garches, France

Pierre Damas, Department of General Intensive Care, University Hospital of Liege, Domaine universitaire de Liège, Liege, Belgium Kristina B. Kristoffersen, Department of Infectious Diseases, Aarhus University Hospital, Aarhus N, Denmark

Carolina F. Oliveira, Department of Internal Medicine, School of Medcine, Federal University of Minas Gerais, Belo Horizonte, Brazil Daiana Stolz and Michael Tamm, Clinic of Pneumology and Pulmonary Cell Research, University Hospital Basel, Basel, Switzerland
Evelien de Jong and Armand R.J. Girbes, Department of Intensive Care, VU University Medical Center, Amsterdam, The Netherlands

Konrad Reinhart, Department of Anesthesiology and Intensive Care Medicine, Jena University Hospital, Jena, Germany; and Clinical Trial Centre Leipzig, University of Leipzig, Leipzig, Germany Yahya Shehabi, Critical Care and Peri-operative Medicine, Monash Health, Melbourne, Australia; and School of Clinical Sciences, Faculty of Medicine Nursing and Health Sciences, Monash University, Melbourne, Australia

Alessia Verduri, Department of Medical and Surgical Sciences,Policlinico di Modena, University of Modena and Reggio Emilia, Modena, Italy

Vandack Nobre, Department of Internal Medicine, School of Medicine, Universidade Federal de Minas Gerais, Belo Horizonte, Minas Gerais, Brazil

Maarten Nijsten, University Medical Centre, University of Groningen, Groningen, The Netherlands

Dylan W. deLange, University Medical Center Utrecht, Utrecht, The Netherlands

Jos A.H. van Oers, Elisabeth Tweesteden Hospital, Tilburg, The Netherlands

Albertus Beishuizen, Medisch Spectrum Twente, Enschede, The Netherlands

Beat Mueller, Medical University Department, Kantonsspital Aarau, Aarau, Switzerland; and Faculty of Medicine, University of Basel, Basel, Switzerland

This work is licensed under the Creative Commons Attribution 4.0 
15-89 [CKD 2-4] and GFR<15 [CKD 5]). The main efficacy and safety endpoints were duration of antibiotic treatment and 30-day mortality.

Results: Mean duration of antibiotic treatment was significantly shorter in PCT-guided $(\mathrm{n}=2,492)$ compared to control patients $(\mathrm{n}=2,510) \quad(9.5-7.6$ days; adjusted difference in days -2.01 [95\% CI, -2.45 to -1.58$]$ ). CKD 5 patients had overall longer treatment durations, but a 2.5-day reduction in treatment duration was still found in patients receiving in PCT-guided care (11.3 vs. 8.6 days [95\% CI -3.59 to -1.40$])$. There were 397 deaths in 2,492 PCT-group patients (15.9\%) compared to 460 deaths in 2,510 control patients (18.3\%) (adjusted odds ratio, 0.88 [95\% CI 0.78 to 0.98)]. Effects of PCT-guidance on antibiotic treatment duration and mortality were similar in subgroups stratified by infection type and clinical setting ( $p$ interaction $>0.05$ ).

Conclusions: This individual patient data meta-analysis confirms that the use of PCT in patients with impaired kidney function, as assessed by admission creatinine levels, is associated with shorter antibiotic courses and lower mortality rates.

Keywords: antibiotic stewardship; chronic kidney disease; procalcitonin.

\section{Introduction}

Antibiotic stewardship has become an international priority to reduce risk of multi-resistant organisms and potential drug-related side effects for patients [1]. Among different other clinical and laboratory markers, procalcitonin (PCT) has emerged as an adjunct to clinical judgement to assess the risk for bacterial infection and treatment response to antibiotic therapy [2-4]. PCT is released by different tissues in the body in response to systemic inflammation caused by bacterial infections through cytokine stimulation (e.g., interleukin [IL)-1 13 , IL-6 or tumor necrosis factor [TNF]- $\alpha$ ) [5-7]. The short time until PCT increases after bacterial infection and the kinetic profile, which differs in patients with and without response to infection [8], make PCT an interesting marker to monitor patients with infections and help to early reduce antibiotic treatment in case of a favorable clinical response $[9,10]$. Multiple randomized studies found that antibiotic stewardship based on clinical judgement and PCT levels results in reduced antibiotic exposure, lower risk for side-effects and improvements in clinical outcomes including overall survival [11-13]. Two patient data meta-analyses published in 2018 with focus on patients with respiratory tract infections [11,14] and patients with sepsis [15] also confirmed associations of PCT-guidance and lower antibiotic consumption as well as improved health benefits. Still it is important to understand that PCT should be used only as an adjunct to clinical decision making since several factors and conditions may cause false positive and false negative results [16].

Kidney function has been identified as an important parameter that influences PCT kinetics. While PCT levels in healthy individuals are below the limit of detection, in patients with chronic kidney disease (CKD), elevated PCT levels and slower PCT kinetics have been reported [17-19]. This may be due to different potential reasons including low grade inflammation in CKD patients with a constant stimulation of PCT $[20,21]$ and a slower clearance of PCT due to impaired renal function. The importance of renal function on PCT kinetics, however, has been controversial with some reports not finding a significant effect of renal clearance on PCT [17, 22, 23], while a recently published case-control study reported a reduced PCT-clearance in patients with impaired kidney function [20]. Importantly, there is a lack of data from clinical trials investigating whether the effectiveness and safety of antibiotic stewardship based on PCT levels is possibly reduced in CKD patients.

Herein, we conducted a secondary analysis using data from an individual-patient data meta-analysis to assess the safety and efficacy of using PCT to guide antibiotic treatment decisions according to kidney function based on admission creatinine levels in patients with respiratory infections or sepsis included in previous randomized trials.

\section{Materials and methods}

\section{Patient population and trial selection}

We conducted a secondary analysis using our updated individual patient database [11, 14, 15, 24-27]. The study selection and data collection was based on the original protocol published in the Cochrane Library [28] and the report was prepared following the Preferred Reporting Items for Systematic Review and Meta-Analysis individual participant data guidelines [29, 30]. Overall, we selected individual patient data from 15 randomized controlled trials including patients with a clinical diagnosis of infection treated in the ICU or in the medical ward and information about kidney function. Hence, in accordance with the initial protocol, we excluded trials lacking information regarding kidney function as well as pediatric trials and those not using PCT for guiding the initiation and duration of antibiotic therapy. 


\section{Trial search and data collection}

For this review the trial search was updated in February 2018 in collaboration with the Cochrane collaboration and conducted in all databases from the date of their inception to February 2018. In addition to the Cochrane Central Register for Controlled Trials (January 2017, Issue 1) databases searched included Medline Ovid (1966 to February 2017) and Embase (1980 to February 2017). All references were screened for eligibility and there were no language or publication restrictions. Two authors (Y.W. and M.A.M.) independently assessed trial eligibility based on titles, abstracts, and full-text reports, with further information being obtained directly from investigators as needed. Study protocols, case report forms, and unedited databases containing individual patient data were requested from investigators of all eligible trials. Data from each trial were first checked against reported results and queries were resolved with the principal investigator, trial data manager, or statistician. Data were assessed in a consistent manner across all trials, with standard definitions and parameters, and thus mortality rates differed slightly from previous reports. In accordance with the Cochrane methodology, we used the Grading of Recommendations, Assessment, Development, and Evaluation (GRADE) [31] approach to assess risk of selection bias, performance bias, detection bias, attrition bias, reporting bias, and other types of bias. Initially, the grading was done by two authors (Y.W. and M.A.M.) and if conflicting, the grading was discussed with another author (P.S.) and within the meta-analysis group.

\section{Patients and endpoints}

In the final analysis we enrolled all patients with a known kidney function and a suspected or proven infection who had been included in a previous trial and were randomized either to PCT-guided care or a control group. There were no exclusion criteria except for lack of information regarding kidney function. The main efficacy endpoint was duration of antibiotic treatment within 30 days. The main safety endpoint was all-cause mortality within 30 days of randomization. In case of a shorter follow-up period, the available information in the trials was used (e.g., mortality at the time of hospital discharge). Secondary outcomes comprised length of hospital stay, length of ICU stay, and the need for kidney replacement therapy. The definition of and indication for renal replacement therapy was based on the definition used in the different trials.

Patients were divided into three groups according to the glomerular filtration rate (GFR) (estimated using the MDRD [Modification of Diet in Renal Disease] study equation) and based on the CKD (Chronic Kidney Disease) classification from the KDIGO (Kidney Disease Improving Global Outcomes) [32]. Patients with a GFR above or equal to $90 \mathrm{ml} / \mathrm{min} / 1.73 \mathrm{~m}^{2}$ were assigned to the group with normal kidney function, patients with a GFR between 15 and $89 \mathrm{ml} / \mathrm{min} /$ $1.73 \mathrm{~m}^{2}$ were assigned to the group with impaired renal function and patients with a GFR lower than $15 \mathrm{ml} / \mathrm{min} / 1.73 \mathrm{~m}^{2}$ were assigned to the group with end stage renal disease.

\section{Statistical analysis}

The statistical approach was similar to the previously published Cochrane Library study protocol [28] with additional stratification according to kidney function. Using multivariate hierarchical logistic regression $[33,34]$ we calculated odds ratios (ORs) and 95\% confidence intervals (CIs) for the primary endpoint mortality. Variables in the multivariate analysis included treatment arm, age, treatment setting (ICU, medical ward) and type of infection. To control for within- and between-trial variability, a "trial" variable was added to the model as a random effect. For continuous and binary secondary endpoints, corresponding linear and logistic regression models were fitted, respectively. Patients were analyzed in groups to which they initially were randomly assigned and thus following the intention to treat principle. Predefined subgroup analyses were performed for type of infection, blood culture results, setting (ICU, medical ward) and the level of organ dysfunction (Sequential Organ Failure Assessment (SOFA)). All statistical analyses were done using STATA version 15.1 (StataCorp. College Station, Texas).

\section{Results}

\section{Findings of systematic research and characteristics of included trials}

A total of 990 records were identified in the initial search, of which 71 were assessed for eligibility and a total of 32 trials were potentially eligible for analysis. Seventeen trials were excluded due to lack of clinical data or information regarding kidney function. Within the remaining 15 trials, we excluded 1,833 from 6,835 individual patients due to missing information regarding creatinine values (Figure 1). Our final analysis thus consists of 5,002 individual patient records included in fifteen randomized controlled trials. The trials were conducted in nine different countries, namely Australia, Belgium, Brazil, Denmark, France, Germany, Italy, The Netherlands and Switzerland (Table 1). Eleven of these trials were multicentric, nine were performed in the ICU and six in the emergency department (ED) and medical ward. The three largest trials were the SAPS trial $(n=1,516)$ [12], the ProHOSP trial $(n=1,359)$ [35] and the SISPICT trial $(n=1,089)$ [36]. The PCT algorithms used in the different trials were similar in concept and focused mainly on optimizing antibiotic tailoring by early stopping of therapy based on low PCT cutoff levels or a decrease in PCT from the peak by $\geq 80 \%$. Adherence rates to PCT protocols were variable and ranged from 44 to $97 \%$.

\section{Baseline characteristics}

Overall, 1,420 patients (28.4\%) had a normal kidney function (CKD 1), 2,512 patients (50.2\%) had an impaired renal function (CKD 2-4) and 1,070 patients (21.4\%) had end stage renal disease (CKD 5). 


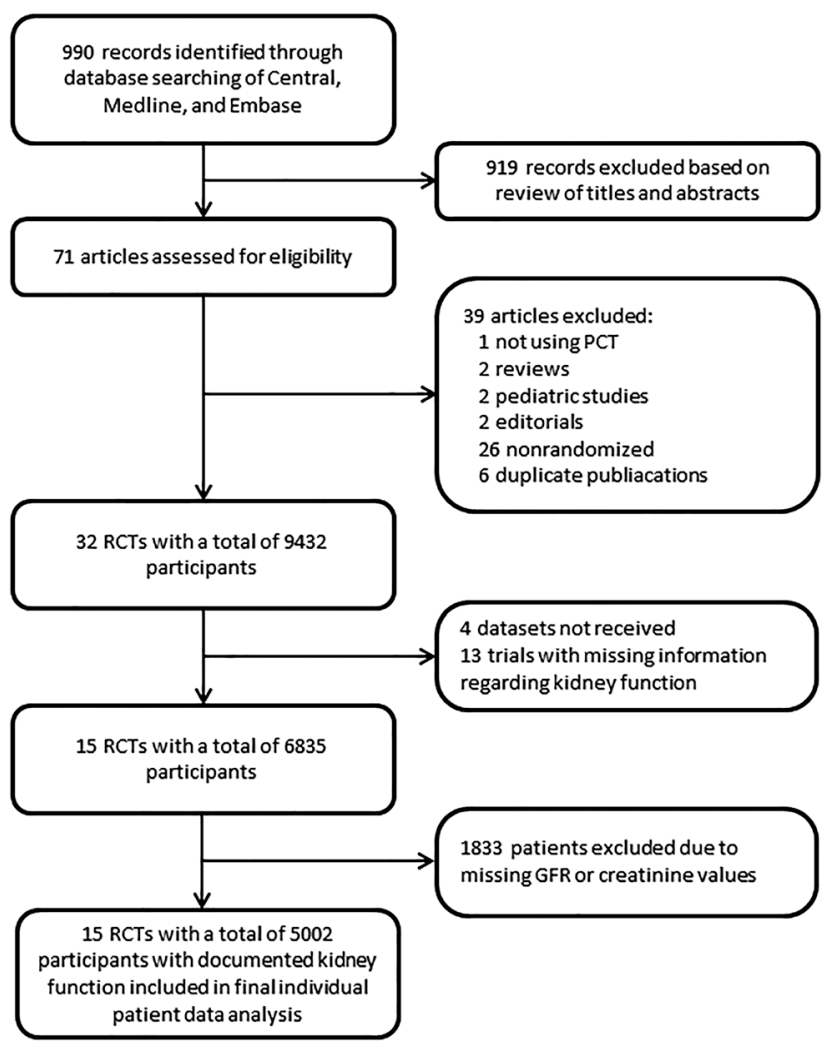

Figure 1: Study flowchart.

$\mathrm{PCT}$, procalcitonin; RCT, randomized controlled trial; GFR, glomerular filtration rate.
Baseline characteristics of patients were overall similar in the PCT and control groups as well as in the different CKD subgroups. Table 2 and Supplementary Material, Appendix Table 1 shows baseline characteristics stratified according to randomization and kidney function. The most common infection focus was the respiratory tract in $29 \%$. The SOFA scores and PCT values on admission were markedly higher in patients with worse kidney function. The majority of patients was treated in the ICU.

\section{Primary efficacy endpoint: duration of antibiotic treatment}

Mean duration of antibiotic treatment was significantly shorter in PCT-guided patients $(\mathrm{n}=2,492)$ compared to control patients $(n=2,510)$ (7.6 vs. 9.5 days, adjusted difference -2.01 days [ $95 \% \mathrm{CI}-2.45$ to 1.58$]$ ). CKD 5 patients had overall longer treatment durations, but a 2.5-day treatment duration reduction was still found in PCT-guided patients (8.6 vs. 11.3 days [ $95 \%$ CI -3.59 to -1.40$]$ ). Effects were similar in subgroups stratified based on type of infection, blood culture result, treatment location (ICU vs. ward) and SOFA score (Figures 2, 3, Supplementary Material, Appendix Tables 2-4 and Appendix Figures 1 and 2).

Table 1: Characteristics of included trials.

\begin{tabular}{|c|c|c|c|c|c|c|c|c|}
\hline $\begin{array}{l}\text { First author, } \\
\text { year }\end{array}$ & Country & $\begin{array}{l}\text { Type of } \\
\text { trial }\end{array}$ & Setting & $\begin{array}{r}\text { Patients } \\
\text { included } \\
\text { in original } \\
\text { trial, } n\end{array}$ & $\begin{array}{r}\text { Patients } \\
\text { excluded, } \\
\text { missing } \\
\text { information } \\
\text { regarding } \\
\text { kidney } \\
\text { function, } n\end{array}$ & Clinical diagnosis & $\begin{array}{l}\text { Type of PCT } \\
\text { algorithm and PCT } \\
\text { cutoffs used, } \mu \mathrm{g} / \mathrm{L}\end{array}$ & $\begin{array}{l}\text { Adherence } \\
\text { to PCT } \\
\text { protocol, \% }\end{array}$ \\
\hline Annane (2013) & France & Multicenter & $\mathrm{ICU}$ & 62 & 52 & $\begin{array}{l}\text { Severe sepsis } \\
\text { without overt } \\
\text { source of infection } \\
\text { and negative } \\
\text { blood culture }\end{array}$ & $\begin{array}{l}\text { Initiation and dura- } \\
\text { tion; } R \text { against } A B \text { : } \\
<0.5(<0.25) \text {; } \text { for } \\
A B:>0.5(>0.5)\end{array}$ & $63 \%$ \\
\hline $\begin{array}{l}\text { Bouadma } \\
\text { (ProRATA, } \\
2010 \text { ) }\end{array}$ & France & Multicenter & ICU & 621 & 593 & $\begin{array}{l}\text { Critically ill pa- } \\
\text { tients with } \\
\text { assumed/proven } \\
\text { bacterial infection }\end{array}$ & $\begin{array}{l}\text { Initiation and dura- } \\
\text { tion; } R \text { against } A B \text { : } \\
<0.5(<0.25) \text {; } R \text { for } \\
A B:>0.5(>0.1)\end{array}$ & $46 \%$ \\
\hline Layios (2012) & Belgium & $\begin{array}{l}\text { Single } \\
\text { center }\end{array}$ & ICU & 379 & 3 & $\begin{array}{l}\text { Critically ill pa- } \\
\text { tients with sus- } \\
\text { pected infection }\end{array}$ & $\begin{array}{l}\text { Initiation; R against } \\
A B:<0.5(<0.25) ; R \\
\text { for } A B:>0.5(>1.0)\end{array}$ & $46.3 \%$ \\
\hline $\begin{array}{l}\text { Krisstoffersen } \\
(2009)\end{array}$ & Denmark & Multicenter & $\begin{array}{l}\text { ED, medical } \\
\text { ward }\end{array}$ & 210 & 208 & $\begin{array}{l}\text { Lower ARI without } \\
\text { radiographic } \\
\text { confirmation }\end{array}$ & $\begin{array}{l}\text { Initiation and dura- } \\
\text { tion; } R \text { against } A B \text { : } \\
<0.25 \text {; } \text { for } A B:>0.25 \\
(>0.5)\end{array}$ & $59 \%$ \\
\hline
\end{tabular}


Table 1: (continued)

\begin{tabular}{|c|c|c|c|c|c|c|c|c|}
\hline $\begin{array}{l}\text { First author, } \\
\text { year }\end{array}$ & Country & $\begin{array}{l}\text { Type of } \\
\text { trial }\end{array}$ & Setting & $\begin{array}{r}\text { Patients } \\
\text { included } \\
\text { in original } \\
\text { trial, } n\end{array}$ & $\begin{array}{r}\text { Patients } \\
\text { excluded, } \\
\text { missing } \\
\text { information } \\
\text { regarding } \\
\text { kidney } \\
\text { function, } n\end{array}$ & Clinical diagnosis & $\begin{array}{l}\text { Type of PCT } \\
\text { algorithm and PCT } \\
\text { cutoffs used, } \mu \mathrm{g} / \mathrm{L}\end{array}$ & $\begin{array}{l}\text { Adherence } \\
\text { to PCT } \\
\text { protocol, \% }\end{array}$ \\
\hline Oliveira (2013) & Brazil & Multicenter & ICU & 94 & 75 & $\begin{array}{l}\text { Severe sepsis or } \\
\text { septic shock (SOFA } \\
\text { score }>10 \text { and/or } \\
\text { bacteremia) }\end{array}$ & $\begin{array}{l}\text { Discontinuation; } \\
\text { initial<1.0: } R \text { against } \\
A B: 0.1 \text { at day } 4 ; \\
\text { initial }>1.0: \text { against: } \\
>90 \% \text { drop over peak } \\
\text { value }\end{array}$ & $87.8 \%$ \\
\hline $\begin{array}{l}\text { Christ-Crain } \\
\text { (ProCAP, } \\
\text { 2006) }\end{array}$ & Switzerland & $\begin{array}{l}\text { Single } \\
\text { center }\end{array}$ & $\begin{array}{l}\text { ED, medical } \\
\text { ward }\end{array}$ & 302 & 5 & $\begin{array}{l}\text { CAP with radio- } \\
\text { graphic } \\
\text { confirmation }\end{array}$ & $\begin{array}{l}\text { Initiation and dura- } \\
\text { tion; } R \text { against } A B \text { : } \\
<0.25(<0.1) \text {; } R \text { for } \\
A B:>0.25(>0.5)\end{array}$ & $87 \%$ \\
\hline $\begin{array}{l}\text { Stolz (Pro- } \\
\text { COLD, 2007) }\end{array}$ & Switzerland & $\begin{array}{l}\text { Single } \\
\text { center }\end{array}$ & $\begin{array}{l}\text { ED, medical } \\
\text { ward }\end{array}$ & 208 & 174 & $\begin{array}{l}\text { COPD exacerba- } \\
\text { tion according to } \\
\text { the global initia- } \\
\text { tive for chronic } \\
\text { obstructive lung } \\
\text { disease guidelines }\end{array}$ & $\begin{array}{l}\text { Initiation and dura- } \\
\text { tion; } R \text { against } A B \text { : } \\
<0.25(0.1) \text {; } R \text { for } A B \text { : } \\
>0.25\end{array}$ & $\begin{array}{l}\text { Not } \\
\text { reported }\end{array}$ \\
\hline $\begin{array}{l}\text { Christ-Crain } \\
\text { (ProRESP, } \\
\text { 2004) }\end{array}$ & Switzerland & $\begin{array}{l}\text { Single } \\
\text { center }\end{array}$ & ED & 243 & 24 & $\begin{array}{l}\text { Lower ARI with } \\
\text { radiographic } \\
\text { confirmation }\end{array}$ & $\begin{array}{l}\text { Initiation; } R \text { against } \\
A B:<0.25(<0.1) ; R \\
\text { for } A B:>0.25(>0.5)\end{array}$ & $83 \%$ \\
\hline $\begin{array}{l}\text { De Jong (SAPS, } \\
2016)\end{array}$ & $\begin{array}{l}\text { The } \\
\text { Netherlands }\end{array}$ & Multicenter & ICU & 1516 & 0 & $\begin{array}{l}\text { Critically ill pa- } \\
\text { tients with pre- } \\
\text { sumed infection }\end{array}$ & $\begin{array}{l}\text { Duration; } R \text { against } \\
A B:<0.5 \text { or }>80 \% \\
\text { drop over peak value }\end{array}$ & $44 \%$ \\
\hline $\begin{array}{l}\text { Bloss (SISPICT } \\
\text { 2016) }\end{array}$ & Germany & Multicenter & $\mathrm{ICU}$ & 1089 & 13 & $\begin{array}{l}\text { Severe sepsis and } \\
\text { septic shock }\end{array}$ & $\begin{array}{l}\text { Discontinuation at } \\
\text { day } 4,7 \text { and } 10 ; R \\
\text { against } A B:<1.0 \text { or } \\
>50 \% \text { drop to previ- } \\
\text { ous value }\end{array}$ & $49.6 \%$ \\
\hline $\begin{array}{l}\text { Shehabi } \\
(2014)\end{array}$ & Australia & Multicenter & $\mathrm{ICU}$ & 394 & 355 & $\begin{array}{l}\text { Suspected sepsis, } \\
\text { undifferentiated } \\
\text { infections }\end{array}$ & $\begin{array}{l}\text { Duration; } R \text { against } \\
A B:<0.25(<0.1) \text { or }> \\
90 \% \text { drop }\end{array}$ & $97 \%$ \\
\hline Verduri (2015) & Italy & Multicenter & $\begin{array}{l}\text { ED, medical } \\
\text { ward }\end{array}$ & 178 & 166 & $\begin{array}{l}\text { Acute COPD } \\
\text { exacerbation }\end{array}$ & $\begin{array}{l}\text { Initiation; } R \text { against } \\
A B:<0.1 ; R \text { for } A B: \\
>0.25\end{array}$ & $\begin{array}{l}\text { Not } \\
\text { reported }\end{array}$ \\
\hline Nobre (2008) & Switzerland & Multicenter & $\mathrm{ICU}$ & 79 & 71 & $\begin{array}{l}\text { Suspected severe } \\
\text { sepsis or septic } \\
\text { shock }\end{array}$ & $\begin{array}{l}\text { Duration; } R \text { against } \\
A B:<0.5(<0.25) \text { or } \\
>80 \% \text { drop; } R \text { for } A B \text { : } \\
>0.5(>1.0)\end{array}$ & $81 \%$ \\
\hline $\begin{array}{l}\text { Schuetz (Pro- } \\
\text { HOSP, 2009) }\end{array}$ & Switzerland & Multicenter & $\begin{array}{l}\text { ED, medical } \\
\text { ward }\end{array}$ & 1359 & 50 & $\begin{array}{l}\text { Lower ARI with } \\
\text { radiographic } \\
\text { confirmation }\end{array}$ & $\begin{array}{l}\text { Initiation and dura- } \\
\text { tion; } R \text { against } A B \text { : } \\
<0.25(<0.1) \text {; } R \text { for } A B \text { : } \\
>0.25(>0.5)\end{array}$ & $91 \%$ \\
\hline $\begin{array}{l}\text { Stolz (ProVAP, } \\
\text { 2009) }\end{array}$ & Switzerland & Multicenter & ICU & 101 & 44 & $\begin{array}{l}\text { VAP when } \\
\text { intubated }>48 \mathrm{~h}\end{array}$ & $\begin{array}{l}\text { Duration; } R \text { against } \\
A B:<0.5(0.25) \text { or } \\
>80 \% \text { drop; } R \text { for } A B: \\
>0.5(>1.0)\end{array}$ & $\begin{array}{l}\text { Not } \\
\text { reported }\end{array}$ \\
\hline
\end{tabular}

ICU, intensive care unit; ED, emergency department; $A B$, antibiotic; ARI, acute respiratory infection; SOFA, Sequential Organ Failure Assessment, COPD, chronic obstructive pulmonary disease; VAP, ventilator-associated pneumonia; PCT, procalcitonin; R, recommendation. 
Table 2: Baseline characteristics of included patients.

\begin{tabular}{|c|c|c|c|c|c|c|}
\hline \multirow[t]{3}{*}{ Parameter } & \multicolumn{2}{|c|}{$\begin{array}{r}\text { Patients with normal kidney } \\
\text { function CKD stage G1 }\end{array}$} & \multicolumn{4}{|c|}{ Patients with impaired kidney function } \\
\hline & \multirow{2}{*}{$\begin{array}{l}\text { Control } \\
(n=698)\end{array}$} & \multirow{2}{*}{$\begin{array}{r}\text { PCT-group } \\
(n=722)\end{array}$} & \multicolumn{2}{|c|}{ CKD stages G2-G4 } & \multicolumn{2}{|r|}{ CKD stage G5 } \\
\hline & & & $\begin{array}{r}\text { Control } \\
(n=1260)\end{array}$ & $\begin{array}{r}\text { PCT-group } \\
(n=1252)\end{array}$ & $\begin{array}{r}\text { Control } \\
(n=552)\end{array}$ & $\begin{array}{r}\text { PCT-group } \\
(n=518)\end{array}$ \\
\hline Age, years (mean \pm SD) & $56.2(16.3)$ & $55.3(17.1)$ & $68.8(14.4)$ & $68.8(13.8)$ & $71.8(13.4)$ & $72.8(12.7)$ \\
\hline Male sex & $422(60.5 \%)$ & $431(59.7 \%)$ & $745(59.1 \%)$ & $768(61.3 \%)$ & $318(57.6 \%)$ & $309(59.7)$ \\
\hline \multicolumn{7}{|l|}{ Infect focus, n (\%) } \\
\hline Respiratory & $273(39.1 \%)$ & $295(40.9 \%)$ & $373(29.6 \%)$ & $355(28.4 \%)$ & $88(15.9 \%)$ & $78(15.1 \%)$ \\
\hline Pneumoniae & $139(19.9 \%)$ & 137 (19.0\%) & $332(26.3 \%)$ & $324(25.9 \%)$ & $184(33.3 \%)$ & $186(35.9 \%)$ \\
\hline COPD/bronchitis & $64(9.2 \%)$ & $61(8.4 \%)$ & $134(10.6 \%)$ & $116(9.3 \%)$ & 78 (14.1\%) & $74(14.3 \%)$ \\
\hline URTI & $5(0.7 \%)$ & $5(0.7 \%)$ & $4(0.3 \%)$ & $3(0.2 \%)$ & - & - \\
\hline Asthma & $1(0.1 \%)$ & $3(0.4 \%)$ & $1(0.1 \%)$ & $4(0.3 \%)$ & - & - \\
\hline Urinary & $16(2.3 \%)$ & $13(1.8 \%)$ & $35(2.8 \%)$ & $31(2.5 \%)$ & 25 (4.5\%) & $22(4.2 \%)$ \\
\hline Abdominal & $56(8.0 \%)$ & $54(7.5 \%)$ & $201(16.0 \%)$ & 199 (15.9\%) & 77 (13.9\%) & $60(11.6 \%)$ \\
\hline Skin/soft tissue & $10(1.4 \%)$ & $7(1.0 \%)$ & $15(1.2 \%)$ & $7(0.6 \%)$ & $8(1.4 \%)$ & $3(0.6 \%)$ \\
\hline Central nervous system & $18(2.6 \%)$ & $14(1.9 \%)$ & $10(0.8 \%)$ & $16(1.3 \%)$ & $1(0.2 \%)$ & $2(0.4 \%)$ \\
\hline Genital/gynecologic & $2(0.3 \%)$ & $0(0.0 \%)$ & $4(0.3 \%)$ & $3(0.2 \%)$ & $2(0.4 \%)$ & $0(0.0 \%)$ \\
\hline Catheter-related & $5(0.7 \%)$ & $2(0.3 \%)$ & $5(0.4 \%)$ & $5(0.4 \%)$ & $0(0.0 \%)$ & $2(0.4 \%)$ \\
\hline Bloodstream & 10 (1.4\%) & $6(0.8 \%)$ & $4(0.3 \%)$ & $4(0.3 \%)$ & 2 (0.4\%) & $4(0.8 \%)$ \\
\hline Others/Undifferentiated focus ${ }^{a}$ & 99 (14.2\%) & $125(17.3 \%)$ & $142(11.3 \%)$ & $185(14.8 \%)$ & $87(15.8 \%)$ & $87(16.8 \%)$ \\
\hline \multicolumn{7}{|l|}{ Setting, n (\%) } \\
\hline $\mathrm{ED} /$ medical ward & $215(30.8 \%)$ & $213(29.5 \%)$ & $485(38.5 \%)$ & $467(37.3 \%)$ & 269 (48.7\%) & $265(51.2 \%)$ \\
\hline ICU & $483(69.2 \%)$ & 509 (70.5\%) & $775(61.5 \%)$ & $785(62.7 \%)$ & $283(51.3 \%)$ & $253(48.8 \%)$ \\
\hline \multicolumn{7}{|l|}{ Vital signs } \\
\hline Temperature, ${ }^{\circ} \mathrm{C}($ mean \pm SD) & $37.9(1.1)$ & $38.0(1.1)$ & $37.8(1.2)$ & $37.9(1.1)$ & $37.8(1.1)$ & $37.9(1.1)$ \\
\hline \multicolumn{7}{|l|}{ Sepsis score } \\
\hline SOFA score (points) & $5.3(3.6)$ & $5.1(3.3)$ & $8.0(3.7)$ & $8.0(3.9)$ & $10.0(3.8)$ & $9.7(4.0)$ \\
\hline \multicolumn{7}{|l|}{ Laboratory assessments } \\
\hline PCT day $0, \mu \mathrm{g} / \mathrm{L}($ mean $\pm \mathrm{SD})$ & $10.3(41.7)$ & $6.1(25.4)$ & $8.9(23.2)$ & $15.7(45.0)$ & $13.4(31.6)$ & $15.9(41.7)$ \\
\hline$<0.1$ & $113(26.0 \%)$ & $138(19.5 \%)$ & $116(16.6 \%)$ & $127(10.8 \%)$ & 35 (7.5\%) & $35(7.2 \%)$ \\
\hline $0.1-0.25$ & 78 (17.9\%) & $157(22.1 \%)$ & $147(21.0 \%)$ & $192(16.3 \%)$ & $72(15.4 \%)$ & $73(15.1 \%)$ \\
\hline $0.25-0.5$ & $41(9.4 \%)$ & $83(11.7 \%)$ & $72(10.3 \%)$ & 117 (9.9\%) & $51(10.9 \%)$ & $57(11.8 \%)$ \\
\hline $0.5-2$ & $75(17.2 \%)$ & $141(19.9 \%)$ & $106(15.2 \%)$ & $213(18.1 \%)$ & $84(17.9 \%)$ & $94(19.4 \%)$ \\
\hline$>2$ & $128(29.4 \%)$ & $190(26.8 \%)$ & $258(36.9 \%)$ & $530(45.0 \%)$ & $226(48.3 \%)$ & $226(46.6 \%)$ \\
\hline CRP day $0, \mathrm{mg} / \mathrm{L}($ mean $\pm \mathrm{SD})$ & $167.2(123.0)$ & $160.2(120.1)$ & $180.2(126.5)$ & $184.3(130.7)$ & $184.2(131.6)$ & $173.4(133.6)$ \\
\hline Creatinine, umol/L (mean \pm SD) & $47.7(24.1)$ & $45.3(24.9)$ & $121.0(56.3)$ & $121.5(54.4)$ & $232.5(165.0)$ & $238.7(173.4)$ \\
\hline \multicolumn{7}{|l|}{ Additional support, n (\%) } \\
\hline Vasopressor use & $352(72.7 \%)$ & $375(73.4 \%)$ & $734(94.7 \%)$ & $732(93.2 \%)$ & $237(85.6 \%)$ & $208(86.0 \%)$ \\
\hline Ventilator support & $386(79.9 \%)$ & 407 (79.6\%) & $634(82.7 \%)$ & $669(85.5 \%)$ & $210(76.1 \%)$ & $187(76.3 \%)$ \\
\hline Renal replacement & $50(7.2 \%)$ & $60(8.3 \%)$ & $133(10.6 \%)$ & 124 (9.9\%) & $118(21.4 \%)$ & $101(19.5 \%)$ \\
\hline
\end{tabular}

${ }^{a}$ Miscellaneous infections like endocarditis, mediastinitis, surgical wound infections or infection without a documented source.

ED, emergency department; ICU, intensive care unit; SD, standard deviation; SOFA, Sequential Organ Failure Assessment; PCT, procalcitonin; CRP, C-reactive protein; COPD, chronic obstructive pulmonary disease; URTI, upper respiratory tract infection; GFR, glomerular filtration rate; CKD, chronic kidney disease; ESRD, end stage renal disease.

\section{Primary safety endpoint: mortality}

Within 30 days there were 397 deaths in 2,492 PCT-guided patients (15.9\%) compared to 460 deaths in 2,510 controlgroup patients (18.3\%), resulting in an adjusted odds ratio for overall mortality of 0.88 ( $95 \% \mathrm{CI}, 0.78-0.98 ; \mathrm{p}=0.023$ ). There was no evidence for effect modification according to CKD group, but patients with CKD stages 2-4 had the highest mortality benefit from PCT-guidance (14.6 vs. 20\%, adjusted OR 0.74 [95\% CI 0.63-0.87, p<0.001). Mortality effects were similar in subgroups stratified based on type of infection, blood culture result, treatment location (ICU vs. ward) and SOFA score (Figures 4, 5, Supplementary Material, Appendix Table 2). 


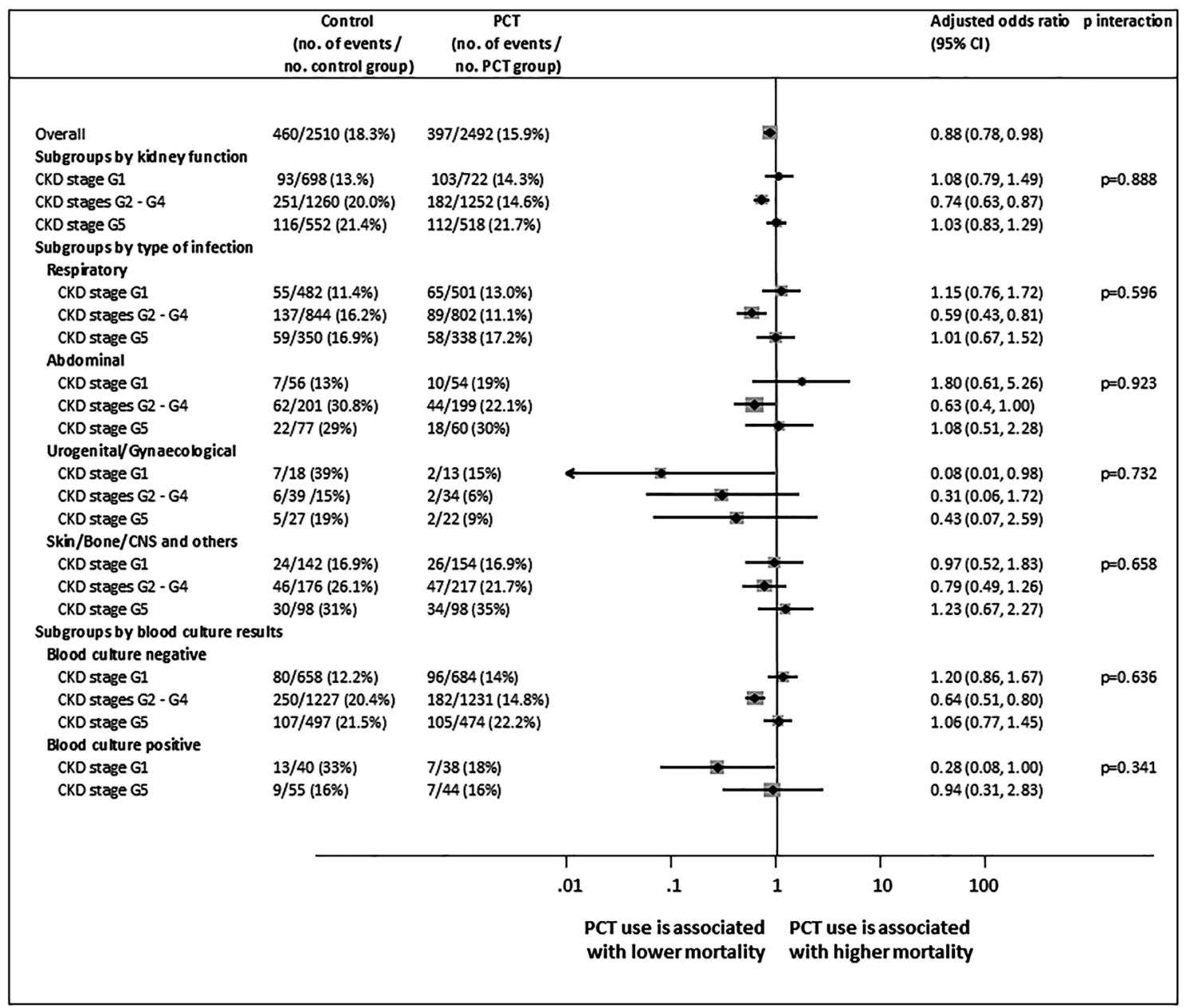

Figure 2: Forest plot showing 30-day mortality. Association of procalcitonin (PCT)-guided antibiotic stewardship and mortality in predefined subgroups.

No., number; $\mathrm{Cl}$, confidence interval; CDK, chronic kidney disease; CNS, central nervous system.

\section{Secondary safety and efficacy endpoints}

\section{Need for renal replacement therapy}

Overall, 285 of 2,492 (11.4\%) patients in the PCT-guided group and 301 of 2,510 (12.0\%) patients in the control group had acute renal failure and needed to undergo dialysis in the course of infection (adjusted OR 0.93 [95\% CI 0.81-1.07], $\mathrm{p}=0.329)$. The risk for replacement therapy increased with CKD stage (7.8\% in CKD 1, 10.2\% in CKD 2-4, and 20.5\% in CKD 5). Consistent with the overall finding, there were also no significant differences in renal replacement therapy rates between PCT-guided and control subjects throughout all subgroup analyses and no evidence of subgroup effect could be observed ( $p$ for interaction $\geq 0.05$, each)
(Supplementary Material, Appendix Tables 3 and 4, Appendix Figures 1 and 2).

\section{Length of stay}

Length of hospital stay as well as length of ICU stay did not significantly differ in the PCT-guided and the control group (overall $22.7 \pm 25.3$ vs. $23.4 \pm 24.1$ days, adjusted difference 0.64 [95\% CI -0.65 to 1.93], $\mathrm{p}=0.329$ and $15.1 \pm 16.5$ vs. $15.4 \pm 17.0$ days, adjusted regression coefficient 0.15 [95\% CI -1.03 to 1.33 ], $\mathrm{p}=0.801$, respectively) (Supplementary Material, Appendix Tables 5 and 6, Appendix Figures 3-6). This finding was similar across all subgroups without evidence for a subgroup effect ( $p$ for interaction $\geq 0.05$, each). 


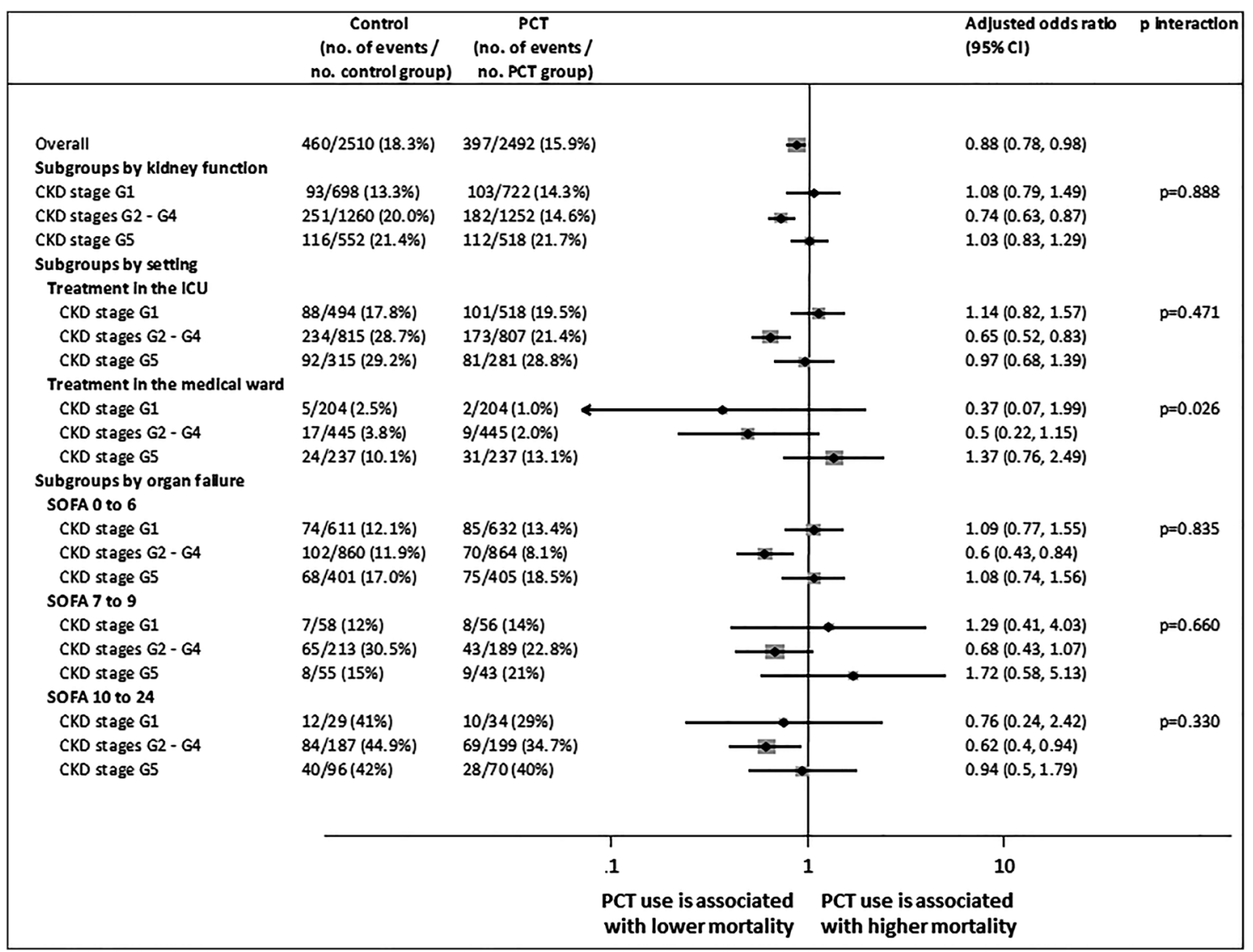

Figure 3: Forest plot showing 30-day mortality. Association of procalcitonin (PCT)-guided antibiotic stewardship and mortality in predefined subgroups.

No., number; $\mathrm{Cl}$, confidence interval; CDK, chronic kidney disease; ICU, intensive care unit; SOFA, Sequential Organ Failure Assessment.

\section{Discussion}

The main findings of this meta-analysis including individual patient data from 5,002 participants from 15 randomized controlled trials are twofold. First, regarding efficacy, we found a significant reduction of antibiotic exposure due to shorter antibiotic treatment durations in PCT-guided patients in all kidney function subgroups. Although, CKD 5 patients had overall longer antibiotic courses, there was still a significant reduction observed in PCT-guided patients compared to the control group. Further, effects were similar in subgroups stratified by type of infection, blood culture results, site of treatment (medical ward vs. ICU), and disease severity according to SOFA score. Second, regarding safety, our overall analysis showed a significant reduction in mortality in PCT-guided patients compared to control group patients with different subgroup analyses showing similar results. Particularly, mortality and other safety outcomes were robust when stratified according to kidney function, by type of infection and by severity of illness.
Consistent with findings of previous studies, we found higher PCT levels in patients with impaired kidney function $[17,18,20,37]$. There are different explanations for the higher PCT levels in patients with CKD. First, persistent low-grade inflammation could contribute to higher PCT baseline levels in CKD patients and impairment in kidney function itself trigger an inflammatory response that elevates PCT levels. Gupta et al. showed that plasma levels of several cytokines, especially IL- $1 \beta$, IL- 6 and TNF- $\alpha$ were increased in patients with impaired kidney function [38]. Hence, these cytokines may also induce expression of PCT by human epithelial cells throughout several tissues in the body. Second, a reduced renal clearance of both, stimulating inflammatory markers and PCT, may increase baseline as well as follow-up PCT levels too. Still, clinical data regarding the effects of renal function on PCT levels have been inconsistent. Meisner and colleagues examined the mechanisms of PCT elimination and revealed that even though the plasma clearance rate seems to be prolonged up to $30-50 \%$ in patients with kidney dysfunction, removal of PCT through the kidneys is not a major component [39]. In 


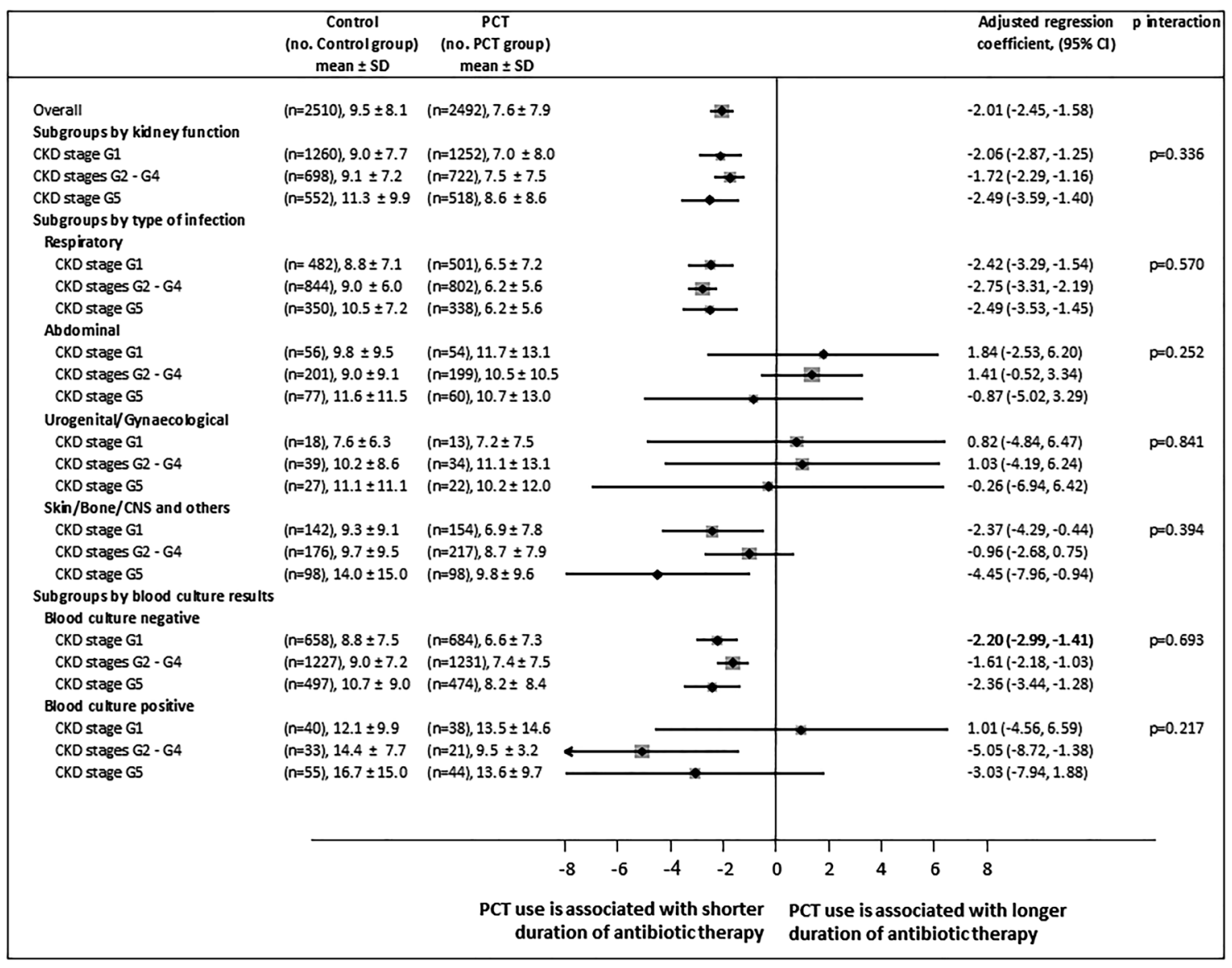

Figure 4: Forest plot showing duration of antibiotic therapy. Association of procalcitonin (PCT)-guided antibiotic stewardship and duration of antibiotic therapy in predefined subgroups.

No., number; SD, standard deviation; $\mathrm{Cl}$, confidence interval; CDK, chronic kidney disease; CNS, central nervous system.

contrast, a recently published case-control study, conducted by $\mathrm{Wu}$ and colleagues, found an association between diminished kidney function and insufficient renal PCT-clearance [20]. They also observed a correlation between cystatin $\mathrm{C}$, a more precise marker of kidney function compared to creatinine, and PCT and argued for similar clearance mechanisms due to similar molecular properties of these two molecules. Our analysis does not provide any new insights regarding mechanisms of PCT-elimination. However, we also confirm higher PCT-levels and longer antibiotic treatment durations in patients with impaired kidney function independent of randomization arm, suggesting that kidney function influences levels of PCT at baseline and during follow-up. Third, patients with CKD may have a functionally declined immune system, resulting in more severe infections. As we know from multiple trials regarding septic patients, early and accurate diagnosis and differentiation from noninfectious causes are crucial for rapidly starting the appropriate initial treatment [40, 41]. This holds also true for patients with CKD and associated comorbidities who are prone to severe infections, sepsis and septic shock. However, overuse of antibiotics in patients with viral instead of bacterial infection as well as unnecessarily long antibiotic duration are jointly responsible for the development of multidrugresistant bacterial pathogens [42, 43]. Importantly, unnecessary administration and prolonged duration of antibiotic treatment contributes to antibiotic side effects and to a possible further impairment of kidney function and thus, worsening clinical outcomes. Hence, once antibiotic treatment is started, daily assessment of patients using clinical and objective parameters such as biomarkers is important. In recent years, PCT has been demonstrated to be a helpful adjunct to clinical judgement and traditional clinical parameters to decide whether to start antibiotic treatment and how long treatment should be continued. PCT levels increase within 6-12 h after bacterial invasion, are highest in patients with bacteremia, correlate with disease severity $[24,44]$ and rapidly fall by about $50 \%$ each day during resolution of infection. In contrast, PCT 


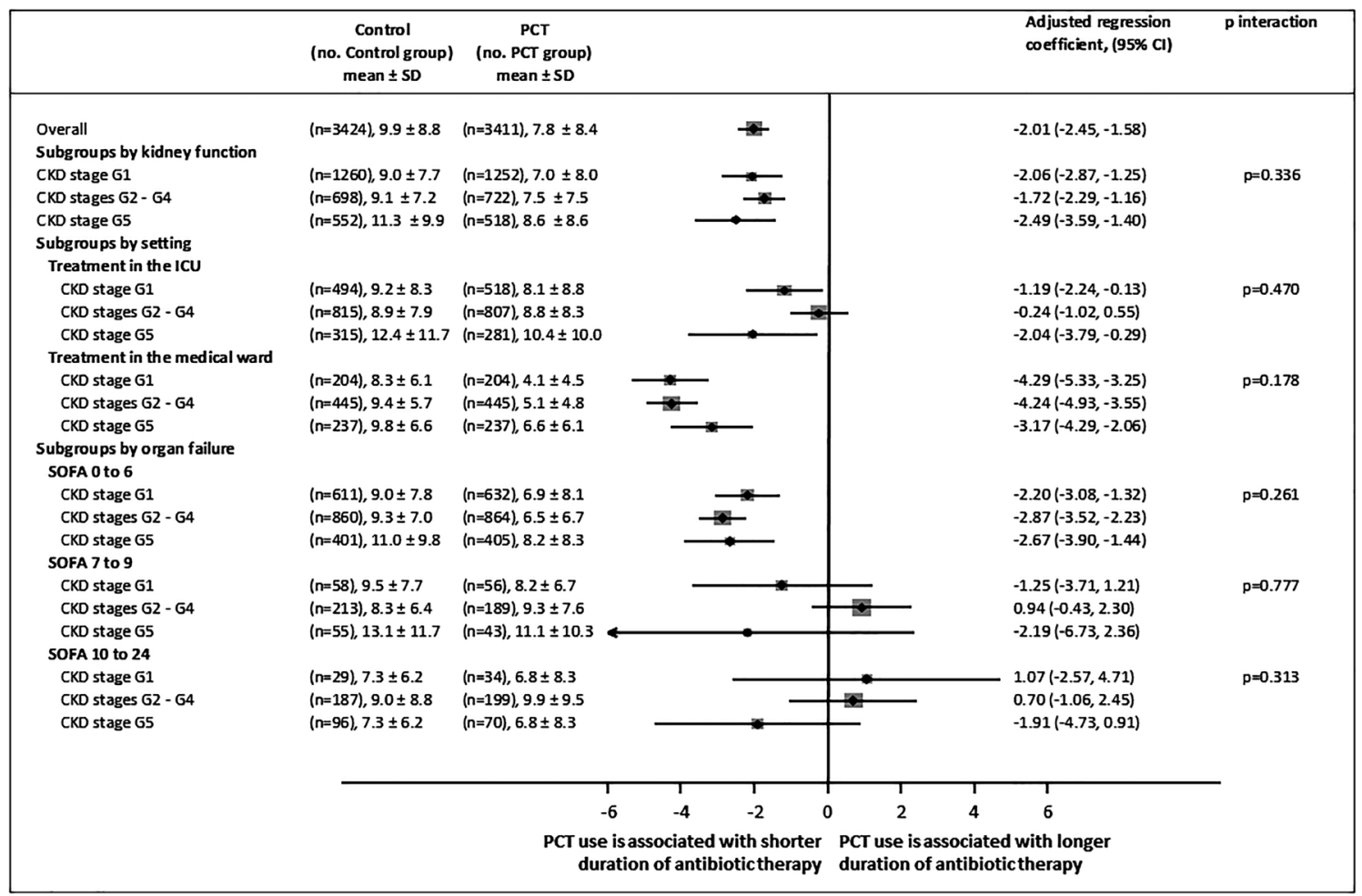

Figure 5: Forest plot showing duration of antibiotic therapy. Association of procalcitonin (PCT)-guided antibiotic stewardship and duration of antibiotic therapy in predefined subgroups.

No., number; SD, standard deviation; Cl, confidence interval; CDK, chronic kidney disease; ICU, intensive care unit; SOFA, Sequential Organ Failure Assessment.

expression is blocked by cytokines, which are typically released in response to viral infection, making it a more specific parameter to differentiate between bacterial and viral infection [5, 45]. Several studies investigating the diagnostic accuracy of PCT yielded diverging results [46, 47]. However, observational studies found sensitivities and specificities of around $80 \%$, which could be increased using algorithms containing a variety of PCT-cutoff points in combination with clinical criteria [3, 48]. Regarding safety of usage of such a PCT-algorithm, two different patient-level meta-analyses of high quality randomized controlled trials on patients with acute respiratory infections and critical illness showed improved survival, when antibiotic treatment was based on PCT-values [11, 15]. In the safety assessment, our study showed no difference in mortality between the PCT-guided and the control group. But the reduced antibiotic treatment durations as well as no obvious difference in need for renal replacement therapy, length of hospital and length of ICU stay, did not reveal any associated harm. Therefore, it is helpful to use PCT to guide antibiotic stewardship in patients with CKD, but due to the finding of significant increased levels of PCT in patients with CKD even without infection, higher cutoff values seem reasonable. The earlier mentioned case-control study by Wu et colleagues revealed a $94.7 \%$ sensitivity and $90.8 \%$ specificity with the use of a PCT cutoff value of $0.075 \mathrm{ng} / \mathrm{mL}$.

Our analysis supports the use of PCT in patients with impaired kidney function and is in line with several previous studies [11, 25, 26]. In PCT-guided patients, antibiotic therapy was shortened by an average of 2 days. This effect tended to be more pronounced in patients with end stage renal disease and those suffering from respiratory tract infections. These effects were not significant in all subgroups due to small sample sizes, but the negative interaction test points to robust results. Clearly, more data is needed to look at specific patient populations, which were underrepresented in our analysis despite the individual data meta-analysis approach.

The strength of this meta-analysis includes a predefined study protocol, a comprehensive search and retrieval of all relevant trials, and a network that permitted inclusion of individual patient data from most eligible trials. We also standardized outcome definitions across trials and performed appropriate subgroup and sensitivity 
analyses, thereby overcoming the limitations of previous meta-analyses with aggregated data to allow more definitive conclusions. To our knowledge, this is the first analysis addressing the effects of PCT-guidance in patients with CKD.

However, there are some limitations of our study. First, we limited our data to immunocompetent adults and patients not being on hemodialysis before inclusion, thereby reducing generalisability of our conclusions to other patient populations. Second, the heterogeneity of our patient population with regard to focus of infection, clinical setting and disease severity, also limits generalisability of results, in particular with regard to the primary endpoint mortality. Third, the adherence to the PCT-protocols among the studies varied widely from 44 to $97 \%$. Overall, adherence rates were better in low-risk populations, whereas the adherence in high-risk patients was lower. This can be explained by the fact, that in case of clinically seriously ill patients, physicians tend to decide rather on clinical findings than on laboratory results. Because of the fact, that low adherence may interfere with the effects of PCT-protocols, it is important that physicians are educated regarding correct and beneficial use of PCT [49]. Finally, we had very limited data on kidney function for individual patients due to the heterogeneity of trials and thus based our analysis on the initial creatinine level. Particularly, we were not able strictly separate acute from chronic conditions and also do not know how kidney function changed over the course of the hospital stay. This data would also provide important information to better understand the influence of kidney function and its recovery on levels of PCT and associated clinical effects regarding antibiotic use and outcome.

\section{Conclusions}

In conclusion, this individual patient data meta-analysis confirms that in patients with impaired kidney function, estimated based on admission creatinine levels, the use of PCT is associated with shorter antibiotic treatment durations without any apparent harm.

Acknowledgments: We thank all researchers and patients involved in the individual trials for sharing their data.

Research funding: This research was supported by the Roche Diagnostics (KSA 2019 001).

Author contributions: All authors have accepted responsibility for the entire content of this manuscript and approved its submission.
Competing interests: PS and BM report receiving grants from Thermofisher, Roche Diagnostics and BioMerieux. CEL received lecture fees from Thermofisher Brahms, BioMérieux. YS has received unrestricted research grants from Thermo-fisher, BioMérieux, Orion Pharma, and Pfizer. MCC, DS and MT have received research support from Thermo-Fisher. All other authors declare that they have no conflicts of interest. The National Institute for Health Research provided a grant for the initial Cochrane analysis. Roche Diagnostics provided an unrestricted research grant for this analysis. The funders of the study had no bearing on study design, data collection, data analysis, data interpretation, or writing of the report. The corresponding author had full access to all the data in the study and had final responsibility for the decision to submit for publication.

Informed consent: Informed consent was obtained from all individuals included in this study.

Ethical approval: All trial included in the meta-analysis enrolled patients providing informed consent and had approval from their Institutional review boards.

Availability of data and material: Data of the individual trials are available upon reasonable request based on the authorization of principal investigators of the trials.

\section{References}

1. NICE antimicrobial stewardship: right drug, dose, and time?. Lancet 2015;386:717.

2. Lee CC, Kwa ALH, Apisarnthanarak A, Feng JY, Gluck EH, Ito A, et al. Procalcitonin (PCT)-guided antibiotic stewardship in Asia-Pacific countries: adaptation based on an expert consensus meeting. Clin Chem Lab Med 2020;58:1983-91.

3. Schuetz P, Beishuizen A, Broyles M, Ferrer R, Gavazzi G, Gluck EH, et al. Procalcitonin (PCT)-guided antibiotic stewardship: an international experts consensus on optimized clinical use. Clin Chem Lab Med 2019;57:1308-18.

4. Neeser O, Branche A, Mueller B, Schuetz P. How to: implement procalcitonin testing in my practice. Clin Microbiol Infect 2019;25: 1226-30.

5. Linscheid P, Seboek D, Schaer DJ, Zulewski H, Keller U, Muller B. Expression and secretion of procalcitonin and calcitonin gene-related peptide by adherent monocytes and by macrophage-activated adipocytes. Crit Care Med 2004;32: 1715-21.

6. Heilmann E, Gregoriano C, Schuetz P. Biomarkers of infection: are they useful in the ICU? Semin Respir Crit Care Med 2019;40:465-75.

7. Schuetz P, Aujesky D, Muller C, Muller B. Biomarker-guided personalised emergency medicine for all - hope for another hype? Swiss Med Wkly 2015;145:w14079.

8. Schuetz P, Birkhahn R, Sherwin R, Jones AE, Singer A, Kline JA, et al. Serial procalcitonin predicts mortality in severe sepsis patients: results from the multicenter procalcitonin MOnitoring SEpsis (MOSES) study. Crit Care Med 2017;45:781-9. 
9. Schuetz P, Koller M, Christ-Crain M, Steyerberg E, Stolz D, Muller C, et al. Predicting mortality with pneumonia severity scores: importance of model recalibration to local settings. Epidemiol Infect 2008;136:1628-37.

10. Dandona P, Nix D, Wilson MF, Aljada A, Love J, Assicot M, et al. Procalcitonin increase after endotoxin injection in normal subjects. J Clin Endocrinol Metab 1994;79:1605-8.

11. Schuetz P, Wirz Y, Sager R, Christ-Crain M, Stolz D, Tamm M, et al. Effect of procalcitonin-guided antibiotic treatment on mortality in acute respiratory infections: a patient level meta-analysis. Lancet Infect Dis 2018;18:95-107.

12. de Jong E, van Oers JA, Beishuizen A, Vos P, Vermeijden WJ, Haas LE, et al. Efficacy and safety of procalcitonin guidance in reducing the duration of antibiotic treatment in critically ill patients: a randomised, controlled, open-label trial. Lancet Infect Dis 2016;16: 819-27.

13. Schuetz P, Balk R, Briel M, Kutz A, Christ-Crain M, Stolz D, et al. Economic evaluation of procalcitonin-guided antibiotic therapy in acute respiratory infections: a US health system perspective. Clin Chem Lab Med 2015;53:583-92.

14. Schuetz P, Wirz Y, Sager R, Christ-Crain M, Stolz D, Tamm M, et al. Procalcitonin to initiate or discontinue antibiotics in acute respiratory tract infections. Cochrane Database Syst Rev 2017;10: CD007498.

15. Wirz Y, Meier MA, Bouadma L, Luyt CE, Wolff M, Chastre J, et al. Effect of procalcitonin-guided antibiotic treatment on clinical outcomes in intensive care unit patients with infection and sepsis patients: a patient-level meta-analysis of randomized trials. Crit Care 2018;22:191.

16. Rodriguez AH, Aviles-Jurado FX, Diaz E, Schuetz P, Trefler SI, SoleViolan J, et al. Procalcitonin (PCT) levels for ruling-out bacterial coinfection in ICU patients with influenza: a CHAID decision-tree analysis. J Infect 2016;72:143-51.

17. Meisner M, Schmidt J, Huttner H, Tschaikowsky K. The natural elimination rate of procalcitonin in patients with normal and impaired renal function. Intensive Care Med 2000;26(Suppl 2): S212-6.

18. Level C, Chauveau P, Delmas Y, Lasseur C, Pelle G, Peuchant E, et al. Procalcitonin: a new marker of inflammation in haemodialysis patients?. Nephrol Dial Transplant 2001;16:980-6.

19. Grace E, Turner RM. Use of procalcitonin in patients with various degrees of chronic kidney disease including renal replacement therapy. Clin Infect Dis 2014;59:1761-7.

20. Wu SC, Liang CX, Zhang YL, Hu WP. Elevated serum procalcitonin level in patients with chronic kidney disease without infection: a case-control study. J Clin Lab Anal 2020;34:e23065.

21. Dumea R, Siriopol D, Hogas S, Mititiuc I, Covic A. Procalcitonin: diagnostic value in systemic infections in chronic kidney disease or renal transplant patients. Int Urol Nephrol 2014;46:461-8.

22. Dahaba AA, Rehak PH, List WF. Procalcitonin and C-reactive protein plasma concentrations in nonseptic uremic patients undergoing hemodialysis. Intensive Care Med 2003;29:579-83.

23. Herget-Rosenthal S, Klein T, Marggraf G, Hirsch T, Jakob HG, Philipp T, et al. Modulation and source of procalcitonin in reduced renal function and renal replacement therapy. Scand J Immunol 2005;61:180-6.

24. Kutz A, Briel M, Christ-Crain M, Stolz D, Bouadma L, Wolff M, et al. Prognostic value of procalcitonin in respiratory tract infections across clinical settings. Crit Care 2015;19:74.
25. Meier MA, Branche A, Neeser OL, Wirz Y, Haubitz S, Bouadma L, et al. Procalcitonin-guided antibiotic treatment in patients with positive blood cultures: a patient-level meta-analysis of randomized trials. Clin Infect Dis 2019;69:388-96.

26. Schuetz P, Briel M, Christ-Crain M, Stolz D, Bouadma L, Wolff $M$, et al. Procalcitonin to guide initiation and duration of antibiotic treatment in acute respiratory infections: an individual patient data meta-analysis. Clin Infect Dis 2012;55: 651-62.

27. Schuetz P, Muller B, Christ-Crain M, Stolz D, Tamm M, Bouadma L, et al. Procalcitonin to initiate or discontinue antibiotics in acute respiratory tract infections. Cochrane Database Syst Rev 2012;10: Cd007498.

28. Schuetz P, Briel M, Christ-Crain M, Wolbers M, Stolz D, Tamm M, et al. Procalcitonin to initiate or withhold antibiotics in acute respiratory tract infections. Cochrane Database Syst Rev 2008. https://doi.org/10.1002/14651858.cd007498.

29. Stewart LA, Clarke M, Rovers M, Riley RD, Simmonds M, Stewart $G$, et al. Preferred reporting Items for systematic review and metaanalyses of individual participant data: the PRISMA-IPD statement. J Am Med Assoc 2015;313:1657-65.

30. Liberati A, Altman DG, Tetzlaff J, Mulrow C, Gotzsche PC, loannidis JP, et al. The PRISMA statement for reporting systematic reviews and meta-analyses of studies that evaluate healthcare interventions: explanation and elaboration. BMJ 2009;339: b2700.

31. Guyatt GH, Oxman AD, Vist GE, Kunz R, Falck-Ytter Y, AlonsoCoello $P$, et al. GRADE: an emerging consensus on rating quality of evidence and strength of recommendations. BMJ 2008;336: 924-6.

32. Levey AS, Eckardt KU, Tsukamoto Y, Levin A, Coresh J, Rossert J, et al. Definition and classification of chronic Kidney disease: $a$ position statement from Kidney disease: improving global outcomes (KDIGO). Kidney Int 2005;67:2089-100.

33. Thompson SG, Turner RM, Warn DE. Multilevel models for metaanalysis, and their application to absolute risk differences. Stat Methods Med Res 2001;10:375-92.

34. Turner RM, Omar RZ, Yang M, Goldstein H, Thompson SG. A multilevel model framework for meta-analysis of clinical trials with binary outcomes. Stat Med 2000;19:3417-32.

35. Schuetz P, Christ-Crain M, Thomann R, Falconnier C, Wolbers M, Widmer I, et al. Effect of procalcitonin-based guidelines vs standard guidelines on antibiotic use in lower respiratory tract infections: the ProHOSP randomized controlled trial. J Am Med Assoc 2009;302:1059-66.

36. Bloos F, Trips E, Nierhaus A, Briegel J, Heyland DK, Jaschinski U, et al. Effect of sodium selenite administration and procalcitoninguided therapy on mortality in patients with severe sepsis or septic shock: a randomized clinical trial. JAMA Intern Med 2016; 176:1266-76.

37. Sun $Y$, Jiang L, Shao X. Predictive value of procalcitonin for diagnosis of infections in patients with chronic kidney disease: a comparison with traditional inflammatory markers C-reactive protein, white blood cell count, and neutrophil percentage. Int Urol Nephrol 2017;49:2205-16.

38. Gupta J, Mitra N, Kanetsky PA, Devaney J, Wing MR, Reilly M, et al. Association between albuminuria, kidney function, and inflammatory biomarker profile in CKD in CRIC. Clin J Am Soc Nephrol 2012;7:1938-46. 
39. Meisner M, Lohs T, Huettemann E, Schmidt J, Hueller M, Reinhart K. The plasma elimination rate and urinary secretion of procalcitonin in patients with normal and impaired renal function. Eur J Anaesthesiol 2001;18:79-87.

40. Rhodes A, Evans LE, Alhazzani W, Levy MM, Antonelli M, Ferrer R, et al. Surviving sepsis Campaign: international guidelines for management of sepsis and septic shock: 2016. Intensive Care Med 2017;43:304-77.

41. Kumar A, Ellis P, Arabi Y, Roberts D, Light B, Parrillo JE, et al. Initiation of inappropriate antimicrobial therapy results in a fivefold reduction of survival in human septic shock. Chest 2009; 136:1237-48.

42. Gonzales R, Steiner JF, Sande MA. Antibiotic prescribing for adults with colds, upper respiratory tract infections, and bronchitis by ambulatory care physicians. J Am Med Assoc 1997; 278:901-4.

43. Lawrence KL, Kollef MH. Antimicrobial stewardship in the intensive care unit: advances and obstacles. Am J Respir Crit Care Med 2009;179:434-8.

44. Schuetz P, Hausfater P, Amin D, Amin A, Haubitz S, Faessler L, et al. Biomarkers from distinct biological pathways improve early risk stratification in medical emergency patients: the multinational, prospective, observational TRIAGE study. Crit Care 2015;19:377.

45. Christ-Crain M, Muller B. Procalcitonin in bacterial infectionshype, hope, more or less? Swiss Med Wkly 2005;135:451-60.

46. Wacker C, Prkno A, Brunkhorst FM, Schlattmann P. Procalcitonin as a diagnostic marker for sepsis: a systematic review and metaanalysis. Lancet Infect Dis 2013;13:426-35.

47. Tang BM, Eslick GD, Craig JC, McLean AS. Accuracy of procalcitonin for sepsis diagnosis in critically ill patients: systematic review and meta-analysis. Lancet Infect Dis 2007;7: 210-7.

48. Schuetz P, Chiappa V, Briel M, Greenwald JL. Procalcitonin algorithms for antibiotic therapy decisions: a systematic review of randomized controlled trials and recommendations for clinical algorithms. Arch Intern Med 2011;171:1322-31.

49. Huang DT, Yealy DM, Filbin MR, Brown AM, Chang CH, Doi Y, et al. Procalcitonin-guided use of antibiotics for lower respiratory tract infection. N Engl J Med 2018;379:236-49.

Supplementary Material: The online version of this article offers supplementary material (https://doi.org/10.1515/cclm-2020-0931). 